\title{
Handgrip strength and mortality in the oldest old population: the Leiden 85-plus study
}

\author{
Carolina H.Y. Ling MD, Diana Taekema MD, Anton J.M. de Craen PhD, \\ Jacobijn Gussekloo MD PhD, Rudi G.J. Westendorp MD PhD, Andrea B. Maier MD PhD
}

Previously published at www.cmaj.ca

\section{ABSTRACT}

Background: Poor muscular strength has been shown to be associated with increased morbidity and mortality in diverse samples of middle-aged and elderly people. However, the oldest old population (i.e., over 85 years) is underrepresented in such studies. Our objective was to assess the association between muscular strength and mortality in the oldest old population.

Methods: We included 555 participants (65\% women) from the Leiden 85-plus study, a prospective populationbased study of all 85-year-old inhabitants of Leiden, Netherlands. We measured the handgrip strength of participants at baseline and again at age 89 years. We collected baseline data on comorbidities, functional status, levels of physical activity, and adjusted for potential confounders. During the follow-up period, we collected data on mortality.

Results: During a follow-up period of 9.5 years (range 8.510.5 years), 444 (80\%) participants died. Risk for all-cause mortality was elevated among participants in the lowest tertile of handgrip strength at age 85 years (hazard ratio [HR] 1.35, 95\% confidence interval [CI] 1.00-1.82, $p=$ $0.047)$ and the lowest two tertiles of handgrip strength at age 89 years (HR 2.04, Cl 1.24-3.35, $p=0.005$ and HR 1.73, Cl 1.11-2.70, $p=0.016$ ). We also observed significantly increased mortality among participants in the tertile with the highest relative loss of handgrip strength over four years (HR 1.72, Cl 1.07-2.77, $p=0.026$ ).

Interpretation: Handgrip strength, a surrogate measurement of overall muscular strength, is a predictor of allcause mortality in the oldest old population and may serve as a convenient tool for prognostication of mortality risk among elderly people. he fastest growing segment of the elderly population is the group older than 85 years, which is classified as the oldest old age group..$^{1,2}$ The average rate of growth of this group is reported to be $3.8 \%$ annually at a global level. By 2050, the oldest old age group will account for one-fifth of all older persons. ${ }^{2}$
Inactivity is a major problem in this age group, owing to an increased prevalence of medical comorbidities and physical disability with age. Age-related stereotypes and misconceptions (e.g., that older people are invariably unhealthy), coupled with a perceived lack of benefits provided by physical activity, can also represent obstacles to exercise among the oldest old population.

The predisposing influence of a sedentary lifestyle on agerelated cardiometabolic diseases (i.e., obesity, type 2 diabetes mellitus, hypertension and coronary artery disease) is well established. Evidence of the protective effects of physical activity against certain cancers, falls and mental health problems is accumulating. ${ }^{3,4}$ Lack of exercise is also a significant risk factor for sarcopenia, ${ }^{5,6}$ a progressive loss of skeletal muscle mass and strength with aging. ${ }^{7}$ Sarcopenia is highly prevalent among those aged 80 years and older, with reported rates exceeding 50\%. ${ }^{8}$ Reduced muscular strength is associated in turn with outcomes such as physical disability, ${ }^{9,10}$ cognitive decline $^{11}$ and mortality. ${ }^{12,13}$

Handgrip strength, a simple bedside tool, has been shown to be a valid surrogate measurement of overall muscular strength. ${ }^{14,15}$ A recent systematic review has shown that low handgrip strength is associated consistently with premature mortality, disability and other health-related complications among various samples of middle-aged and older people. ${ }^{16}$ Despite its prognostic value, handgrip dynamometry is rarely used in routine geriatric assessment. Epidemiologic studies evaluating the relation in the population of the oldest old are also lacking. We tested the association between handgrip strength and mortality in a prospective population-based study of the oldest old age group. We obtained approval for our study from the Medical Ethical Committee of the Leiden University Medical Center, and informed consent from all participants.

From the Department of Gerontology and Geriatrics (Ling, Taekema, de Craen, Westendorp, Maier); the Department of Public Health and Primary Care (Gussekloo); the Netherlands Consortium for Healthy Aging (de Craen, Westendorp, Maier), Leiden University Medical Center, Leiden, Netherlands; and the Department of Geriatric Medicine (Taekema), Alysis Zorggroep Rijnstate Hospital, Arnhem, Netherlands

CMAJ 2010. DOI:10.1503/cmaj.091278 


\section{Methods}

\section{Participants}

We obtained data from the prospective population-based Leiden 85-plus study, which involved all 85-year-old inhabitants of Leiden, The Netherlands. A total of 599 participants had been enrolled in the study (with a response rate of $87 \%$ ) between September 1997 and September 1999. No selection criteria had been imposed for health status or demographic characteristics. Participants had been visited annually at their homes, where face-to-face interviews, blood samples and various functional tests were performed. Further information on the design of the Leiden 85-plus study and characteristics of its cohort is published elsewhere. ${ }^{17}$

In our analysis, we included 555 participants from whom we obtained reliable handgrip strength measurement at baseline (i.e., age 85 years). We performed a repeat measurement of handgrip strength in a total of 304 participants (55\%) at 89 years of age.

\section{Study design}

\section{Strength assessment}

We measured grip strength to the nearest kilogram of each participant's dominant hand using a Jamar hand dynamometer (Sammons Preston Inc., Bolingbrook, IL). We performed the measurement with the participant in an upright position and with the arm of the measured hand unsupported and parallel to the body. The width of the dynamometer's handle was adjusted to each participant's hand size so that the middle phalanges rested on the inner handle. We instructed participants to exert maximal force. For each participant, we allowed one test trial, then took three test measurements. We used the highest of the three recorded measurements in our analysis. We used the same protocol for each participant's follow-up visit at age 89 years.

To calculate the relative change in handgrip strength over four years, we divided the difference between the handgrip strength measurements taken at ages 85 and 89 years by the baseline value and multiplied the result by 100 . We stratified the measurements of handgrip strength at ages 85 and 89 years and relative change in handgrip strength over four years into tertiles for men and women separately. We then created sex-specific tertiles by combining categories of tertiles for men and for women.

\section{Potential confounders}

We considered sex, anthropometric data, comorbidities, total number of prescription medications and smoking status to be potential confounders of an association between handgrip strength and mortality, and included these data in our analysis. Our measurement of body surface area was based on the Mosteller formula..$^{20} \mathrm{We}$ recorded data on seven medical comorbidities, which were cardiovascular disease (comprising ischemic heart disease, cerebrovascular disease and peripheral vascular disease), hypertension, diabetes mellitus, chronic obstructive pulmonary disease, neoplasm, Parkinson disease, and arthritis (comprising osteoarthritis, rheumatoid arthritis and polymyalgia rheumatica).
We also considered scores on the Mini-Mental State Examination, Geriatric Depression Scale, Activities of Daily Living disability scale, Instrumental Activities of Daily Living disability scale and level of physical activity to represent potential confounders. We measured scores on the Activities of Daily Living and Instrumental Activities of Daily Living disability scales using the Groningen Activity Restriction Scale, where a score of 18 indicates not disabled and a score of 72 indicates severely disabled..$^{18}$

To measure levels of physical exercise beyond routine, daily physical activity, we used four items from the TimeSpending Patterns questionnaire ${ }^{19}$ related to walking for fun, cycling for fun, exercising (either alone or in groups) or other physical activity, and working in the garden. We scored each item from 0 (no activity) to 4 (daily participation in the activity), then added these values together to reach a sum score of physical activity level for each participant.

We also collected data on the living arrangements of participants (i.e., independent or in residential care). We did not include these data in the final model because living arrangement is determined primarily by factors already included in the multivariate model (i.e., functional ablities, cognition, cormorbidities).

\section{Follow-up}

We collected data on mortality among participants until February 2008. The range of the follow-up period was 8.5-10.5 years. Information on follow-up for mortality was available for the whole study population. We obtained dates of deaths from the Dutch civic registry and specific data on causes of death from Statistics Netherlands, which assigns codes for all national death certificates according to the International Classification of Diseases and Related Disorders, 10th revision (ICD-10). Causes of death were divided into cardiovascular causes (ICD-10 codes I00-I99) and non-cardiovascular causes (all other ICD-10 codes). Cardiovascular causes were further classified into coronary artery disease, stroke and others. The non-cardiovascular causes were classified into infection with or without sepsis, pneumonia, neoplasm and others. Pneumonia was included because it is one of the major causes of death among elderly people.

\section{Statistical analysis}

Continuous variables with Gaussian distribution are presented as mean (standard deviation [SD]) and those with non-Gaussian distribution as median (interquartile range [IQR]). We used a paired $t$ test to compare two groups of paired data that were of Gaussian distribution. To adjust for difference in physical capacity between men and women, we created sexspecific tertiles of handgrip strength, which were combined categories of tertiles for men and for women. We used a $\chi^{2}$ test to compare categoric variables and a Kruskal-Wallis test to compare non-normally distributed variables between the tertiles of handgrip strength.

We applied Kaplan-Meier curves to display survival according to tertiles of handgrip strength for participants at age 85 years and for those at age 89 years, and for the relative change in handgrip strength over four years. We assessed the analysis of survival using Cox regression analyses. We 
adjusted the hazard ratios (HR) of mortality for potential confounders using baseline data reflecting presence of comorbidities, total number of prescription medications and smoking status, and data collected at baseline or age 89 years for body surface area, for scores on the Mini-Mental State Examination, the Geriatric Depression Scale, the Activities of Daily Living disability scale and the Instrumental Activities of Daily Living disability scale, and for level of physical activity.

We corrected the HRs for relative change in handgrip strength using data on comorbidities, total number of prescription medications and smoking status at baseline, on body surface area at age 89 years, and on absolute change in scores on the Mini-Mental State Examination, Geriatric Depression Scale, Activities of Daily Living disability scale, Instrumental Activities of Daily Living disability scale, and in level of physical activity over four years. In addition, we analyzed handgrip strength as a continuous variable in the multivariable model with adjustment for the above variables. For all fully adjusted models, we calculated the coefficient of determination (i.e., $R^{2}$ ) using the method of Nagelkerke. ${ }^{21}$ We considered a two-tailed $p$-value of less than 0.05 to be significant.

\section{Results}

The baseline clinical characteristics of the 555 participants in the study according to tertile of handgrip strength are presented in Table 1. Cardiovascular disease was the most prevalent comorbidity. At baseline, the median Mini-Mental State Examination score was 26, with evidence of severe cognitive impairment (i.e., score < 19) in 13\% of participants. At age 89 years, impairment was evident in $25 \%$. The median score on

Table 1: Clinical characteristics at baseline (age 85 years) of the study population by sex-specific tertile of handgrip strength

\begin{tabular}{|c|c|c|c|c|c|}
\hline \multirow[b]{2}{*}{ Variable } & \multirow[b]{2}{*}{$\begin{array}{l}\text { Total population } \\
\qquad n=555\end{array}$} & \multicolumn{3}{|c|}{ Sex-specific tertile of handgrip strength* } & \multirow[b]{2}{*}{$p$ value } \\
\hline & & $\begin{array}{c}\text { Low } \\
n=184\end{array}$ & $\begin{array}{l}\text { Middle } \\
n=177\end{array}$ & $\begin{array}{c}\text { High } \\
n=194\end{array}$ & \\
\hline Sex, female, \% & 65.0 & 64.7 & 64.4 & 66.0 & 0.94 \\
\hline Living arrangement, \% & & & & & $<0.001$ \\
\hline Independent living & 85.9 & 73.9 & 88.7 & 94.8 & \\
\hline Residential care & 14.1 & 26.1 & 11.3 & 5.2 & \\
\hline Smoking status (\%) & & & & & 0.53 \\
\hline Nonsmoker & 50.4 & 55.2 & 46.3 & 49.5 & \\
\hline Previous smoker & 34.1 & 30.1 & 36.7 & 35.6 & \\
\hline Current smoker & 15.5 & 14.8 & 16.9 & 14.9 & \\
\hline \multicolumn{6}{|l|}{ Comorbidity, \% } \\
\hline Cardiovascular disease & 62.5 & 66.8 & 66.1 & 55.2 & 0.031 \\
\hline Hypertension & 38.7 & 33.2 & 41.8 & 41.2 & 0.16 \\
\hline Diabetes mellitus & 15.3 & 18.5 & 15.8 & 11.9 & 0.20 \\
\hline $\begin{array}{l}\text { Chronic obstructive pulmonary } \\
\text { disease }\end{array}$ & 11.7 & 13.0 & 10.7 & 11.3 & 0.78 \\
\hline History of neoplasm & 17.9 & 18.8 & 14.1 & 20.6 & 0.94 \\
\hline Parkinson disease & 2.0 & 2.2 & 1.7 & 2.1 & 0.25 \\
\hline Arthritis & 32.7 & 30.4 & 35.8 & 32.1 & 0.54 \\
\hline $\begin{array}{l}\text { Total no. of prescription medications } \\
{[n=526] \text {, median (IQR) }}\end{array}$ & $2(1-4)$ & $2(1-4)$ & $2(1-4)$ & $2(1-4)$ & 0.07 \\
\hline \multicolumn{6}{|l|}{ Mental status, median (IQR) } \\
\hline MMSE score & $26(23-28)$ & $24(18-27)$ & $27(24-28)$ & $27(25-29)$ & $<0.001$ \\
\hline GDS-15 score $[n=484]$ & $2(1-3)$ & $2(1-4)$ & $2(1-3)$ & $1(0-2)$ & $<0.001$ \\
\hline \multicolumn{6}{|l|}{ Functional status, median (IQR) } \\
\hline ADL disability score $[n=553]$ & $10(9-13)$ & $11(9-17)$ & $10(9-12)$ & $9(9-10)$ & $<0.001$ \\
\hline IADL disability score $[n=553]$ & $17(12-25)$ & $24(16-32)$ & $17(13-22)$ & $14(10-19)$ & $<0.001$ \\
\hline GARS score $[n=553]$ & $27(21-37)$ & $36(25-48)$ & $27(22-34)$ & $24(20-30)$ & $<0.001$ \\
\hline PAS, median (IQR) $[n=538]$ & $7(5-8)$ & $6(4-8)$ & $7(4-8)$ & $7(6-9)$ & $<0.001$ \\
\hline
\end{tabular}

Note: $A D L=$ Activities of Daily Living (score range 9-36), GARS = Groningen Activity Restriction Scale (score range 18-72), GDS-15 = Geriatric Depression Scale-15 (score range 0-15), IADL = Instrumental Activities of Daily Living (score range 9-36), IQR = interquartile range, MMSE = Mini-Mental State Examination (score range 0-30), PAS = Physical Activity Score (sum score of four physically active items on the Time Spending Pattern questionnaire; score range 0-16).

*Reference range for sex-specific handgrip strength tertiles: low (women: 1-16 kg; men: 10-27 kg), middle (women: 17-20 kg; men: $28-33 \mathrm{~kg}$ ), high (women: 21-31 kg; men: 34-54 kg). 
the Geriatric Depression Scale at baseline was 2 (IQR 1-3), with evidence of depression (i.e., score $\geq 4$ ) in 113 participants, who represented $23.4 \%$ of those without severe cognitive impairment at baseline compared with $27.7 \%$ at age 89 years. At baseline, the median score on the Activities of Daily Living disability scale was 10 (IQR 9-13); on the Instrumental Activities of Daily Living disability scale it was 17 (IQR 12-25); and on the Groningen Activity Restriction Scale it was 27 (IQR 21-37). The median sum scores for level of physical activity at baseline was 7 (IQR 5-8).

Lower handgrip strength was significantly related to higher rates of cardiovascular disease $(p=0.031)$ but not to other comorbidities. Lower handgrip strength was also significantly associated $(p<0.001)$ with lower scores on the Mini-Mental State Examination and with higher scores on the Geriatric Depression Scale, the Activities of Daily Living disability scale and the Instrumental Activities of Daily Living disability scale, and, finally, with a lower score for level of physical activity. We also observed a significant difference in living arrangement among participants in different tertiles of handgrip strength, with a greater proportion of participants with lower handgrip strength living in residential care.

Anthropometric data and measurements of handgrip strength collected at ages 85 and 89 years and stratified by sex are presented in Table 2. Of the 555 participants, 357 (64.3\%) lived to age 89 years. Measurements of handgrip strength were not available for 53 participants at age 89 years, most of whom withdrew from the study. Participants who were lost to followup measurement of handgrip strength did not differ significantly $(p \geq 0.083)$ from the group as a whole in comorbidities, cognitive status and functional status at baseline.

Handgrip strength among men was consistently higher than among women, both at baseline (30.6 kg [SD 8.2] v. $18.7 \mathrm{~kg}$ [SD 5.5], $p<0.001)$ and at age 89 years $(25.6 \mathrm{~kg}$ [SD $7.8]$ v. $16.4 \mathrm{~kg}$ [SD 5.0], $p<0.001$ ). Among both women and men, handgrip strength declined significantly from age 85 to 89 years, with an average loss of strength of $1.53 \mathrm{~kg}$ per year among men and $0.85 \mathrm{~kg}$ per year among women. However, no significant difference was evident between men and women in relative loss of handgrip strength over four years $(-19.2 \%$ [SD 16.7] v. $-15.9 \%$ [SD 22.4], $p=0.156)$.

During a follow-up period of 9.5 years (range 8.5-10.5 years), 444 deaths ( $80 \%$ ) occurred, with a greater proportion of deaths among men, at $88.1 \%$, compared with women, at $75.6 \%(p<0.001)$. Mean lifespan was 89.7 years (SD 3.0) among men and 91.0 years (SD 3.0) among women. Of the deaths, $36.7 \%$ had cardiovascular causes (coronary artery disease $30.1 \%$, stroke $26.4 \%$, unspecified $43.5 \%$ ). Of the deaths that were attributed to noncardiovascular causes, $28.8 \%$ were caused by neoplasm, $10.0 \%$ were caused by infection (of which $85.7 \%$ were related to pneumonia) and $61.2 \%$ were unspecified.

Kaplan-Meier cumulative survival curves for all-cause mortality are presented in Figure 1. The curves are stratified by sex-specific tertile of handgrip strength at baseline, of handgrip strength at 89 years of age, and of relative change in handgrip strength over four years. The unadjusted and adjusted HRs for all-cause mortality are shown in Table 3. The HRs are stratified by tertile of handgrip strength at age 85 years, handgrip strength at age 89 years and relative change in handgrip strength from age 85 to 89 years.

After adjusting for possible confounders, we found a significant elevation in risk for all-cause mortality among participants in the lowest tertile of handgrip strength at age 85 years (HR 1.35, 95\% CI 1.00-1.82, $p=0.047$ ) and the lowest two tertiles of handgrip strength at age 89 years (HR 2.04, 95\% CI $1.24-3.35, p=0.005$ and HR 1.73, CI 1.11-2.70, $p=0.016$ ), with greater HRs in the latter age group. We also observed a significantly increased mortality among participants in the tertile with the highest relative loss of handgrip strength over four years (HR 1.72, CI 1.07-2.77, $p=0.026$ ). The coeffi-

Table 2: Handgrip strength and anthropometrical measurements at baseline (age 85 years) and at age 89 years, by sex

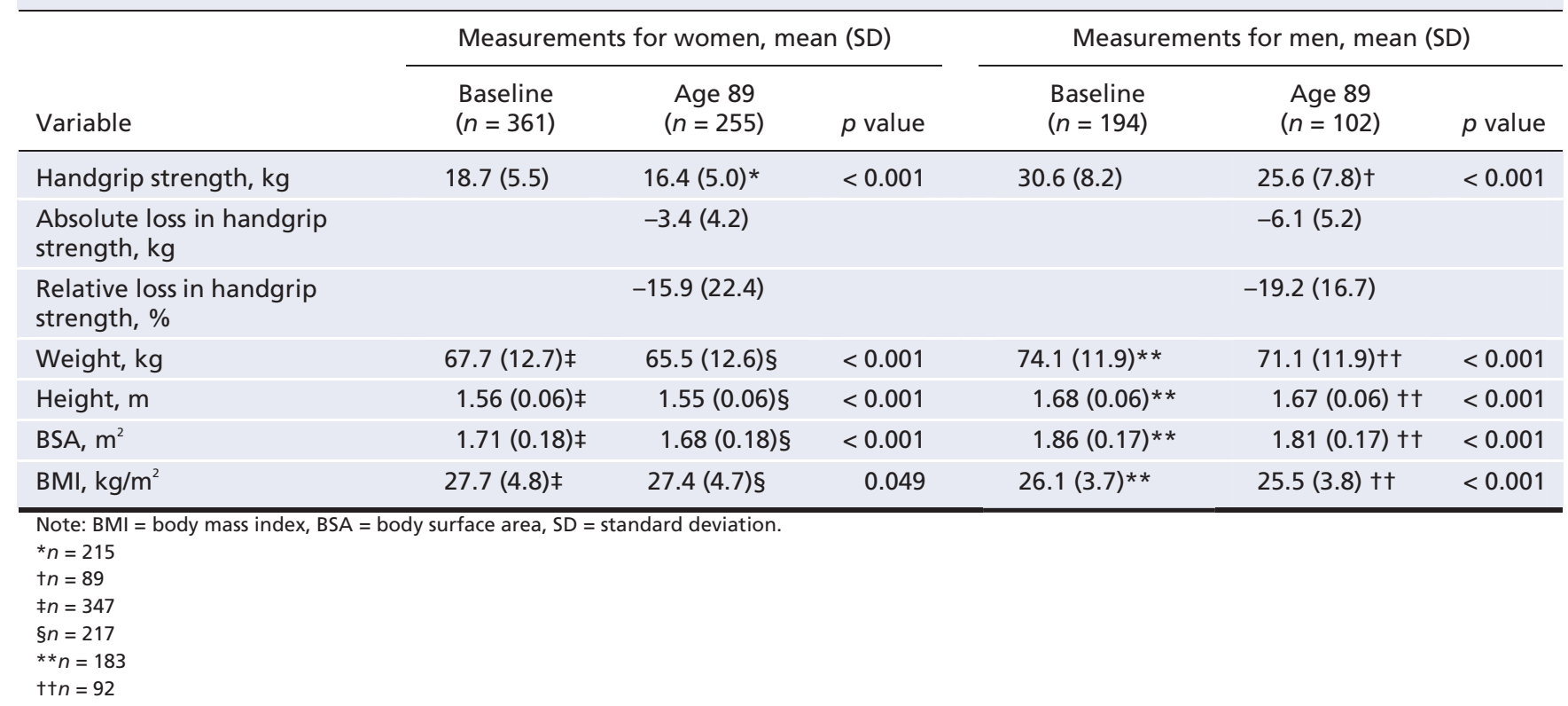


cients of determination were 0.18 for handgrip strength at baseline, 0.27 for handgrip strength at age 89 years and 0.23 for relative loss of handgrip strength. Baseline handgrip strength, handgrip strength at age 89 years and relative loss of handgrip strength were not associated with increased risk for mortality from specific causes.

When we analyzed handgrip strength as a continuous variable in our multivariable analysis, we found that, for each 5$\mathrm{kg}$ reduction in handgrip strength, an increase in risk for allcause mortality occurred at age 85 years (HR 1.11, CI
$1.01-1.23, p=0.040)$ and at age 89 years (HR 1.24, CI 1.04$1.48, p=0.019$ ). Similarly, we observed an increase in risk for all-cause mortality for every additional $5 \%$ loss of relative handgrip strength (HR 1.06, CI 1.01-1.12, $p=0.033$ ).

\section{Interpretation}

Our results show that low handgrip strength at ages 85 and 89 years, and a greater decline in strength over time, are associated with increased all-cause mortality. Our findings also sug-

\section{A: Age 85 years}

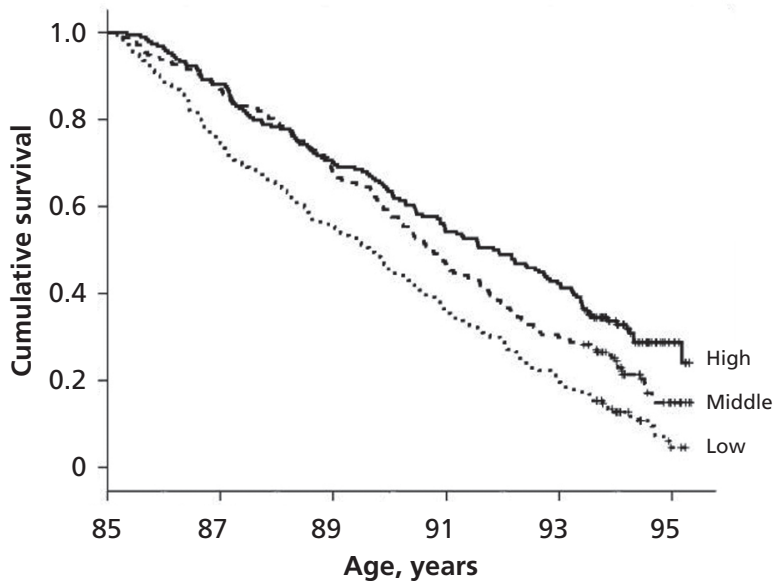

Tertile, $n$ :

$\begin{array}{lrrrrrr}\text { High } & 194 & 171 & 136 & 105 & 82 & 61 \\ \text { Middle } & 177 & 154 & 119 & 82 & 53 & 34 \\ \text { Low } & 184 & 137 & 102 & 65 & 36 & 17\end{array}$

\section{C: Relative change, $85-89$ years}

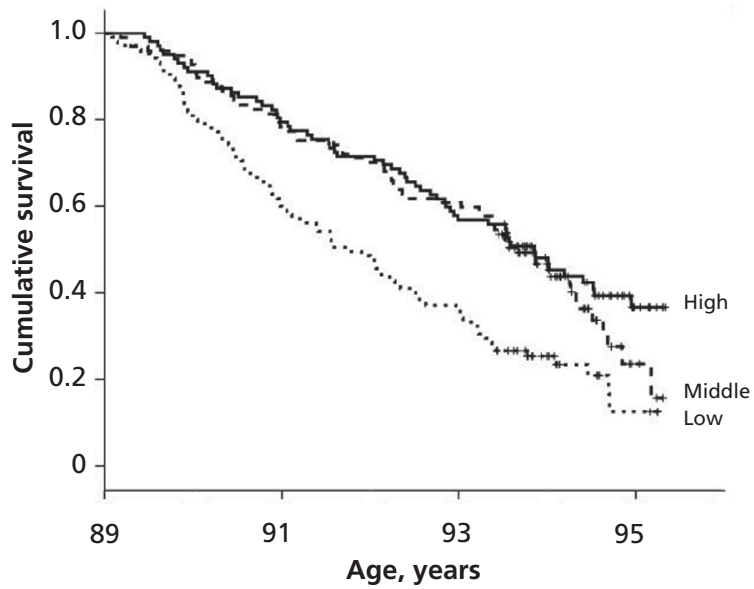

\section{B: Age 89 years}

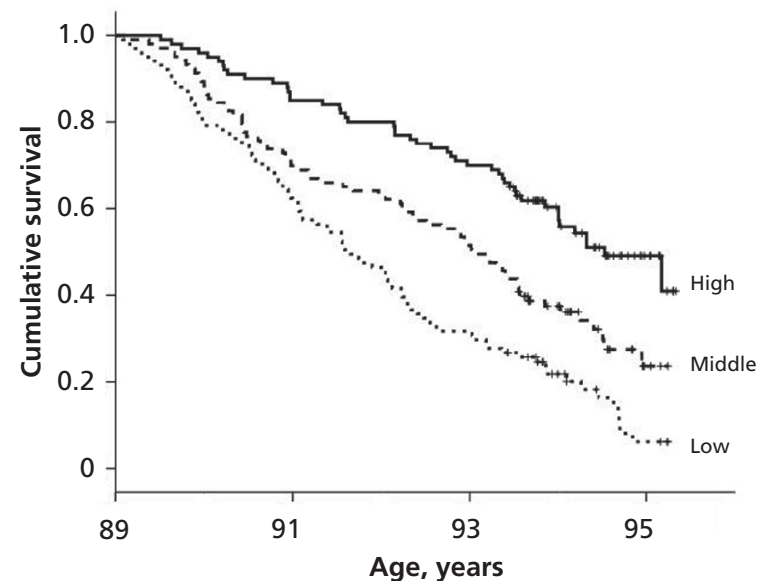

Tertile, $n$ :

$\begin{array}{lllll}\text { High } & 100 & 85 & 70 & 54 \\ \text { Middle } & 103 & 72 & 53 & 33 \\ \text { Low } & 101 & 62 & 32 & 15\end{array}$

Tertile, $n$ :

$\begin{array}{lrlll}\text { High } & 102 & 81 & 58 & 43 \\ \text { Middle } & 97 & 76 & 59 & 36 \\ \text { Low } & 105 & 62 & 38 & 23\end{array}$

Tertile of handgrip strength High

Middle - - - - -

Low

Figure 1: Kaplan-Meier cumulative survival curves for all-cause mortality based on sex-specific tertile of handgrip strength (A) at age 85 years, (B) at age 89 years and (C) by relative change in handgrip strength over four years. The highest tertile of relative change in handgrip strength refers to the tertile with the lowest loss of handgrip strength over four years. 


\begin{tabular}{|c|c|c|c|c|}
\hline \multirow[b]{2}{*}{ Tertile (range, women/men) } & \multicolumn{2}{|r|}{ Unadjusted } & \multicolumn{2}{|r|}{ Adjusted* } \\
\hline & $n$ & $\mathrm{HR}(95 \% \mathrm{Cl})$ & $n$ & $\mathrm{HR}(95 \% \mathrm{Cl})$ \\
\hline \multicolumn{5}{|l|}{ Age 85 years, kg } \\
\hline Low $\quad(1-16) /(10-27)$ & 184 & $1.87(1.48-2.34)$ & 115 & $1.35(1.00-1.82)$ \\
\hline Middle $(17-20) /(28-33)$ & 177 & $1.30(1.03-1.65)$ & 153 & $1.24(0.95-1.62)$ \\
\hline High $\quad(21-31) /(34-54)$ & 194 & 1.00 (ref) & 170 & 1.00 (ref) \\
\hline \multicolumn{5}{|l|}{ Age 89 years, kg } \\
\hline$(2-14) / 6-21)$ & 101 & $3.10(2.16-4.44)$ & 55 & $2.04(1.24-3.35)$ \\
\hline Middle $(15-18) /(22-28)$ & 103 & $1.90(1.31-2.75)$ & 80 & $1.73(1.11-2.70)$ \\
\hline High $\quad(19-31) /(29-48)$ & 100 & 1.00 (ref) & 90 & 1.00 (ref) \\
\hline \multicolumn{5}{|l|}{$\begin{array}{l}\text { Relative change in handgrip strength } \\
\text { over time, } \% \dagger\end{array}$} \\
\hline Low $\quad(-87.5$ to -25.0$) /(-76.9$ to -26.3$)$ & 105 & $2.07(1.47-2.90)$ & 58 & $1.72(1.07-2.77)$ \\
\hline Middle $(-23.8$ to -6.7$) /(-25.6$ to -13.8$)$ & 97 & $1.18(0.82-1.68)$ & 82 & $1.29(0.81-2.04)$ \\
\hline$(-6.25$ to 90.0$) /(-13.6$ to 20.0$)$ & 102 & 1.00 (ref) & 84 & 1.00 (ref) \\
\hline
\end{tabular}

Note: $\mathrm{Cl}=$ confidence interval, $\mathrm{HR}=$ hazard ratio, ref = reference group.

* Hazard ratios for handgrip strength at ages 85 and 89 years were corrected for baseline data for comorbidities, total number of prescription medications and smoking status, and data at baseline or age 89 years for body surface area, for scores on the MiniMental State Examination, the Geriatric Depression Scale, the Activities of Daily Living disability scale and the Instrumental Activities of Daily Living disability scale, and for level of physical activity. Hazard ratios for relative change in handgrip strength were corrected for comorbidities, total number of prescription medications and smoking status at baseline, body surface area at age 89 years, and absolute change in scores on the Mini-Mental State Examination, Geriatric Depression Scale, Activities of Daily Living disability scale, Instrumental Activities of Daily Living disability scale, and level of physical activity over four years.

tThe highest tertile (i.e., reference tertile) refers to that showing the least loss of handgrip strength over four years.

gest a greater association between handgrip strength and mortality with increasing age.

The mechanisms underlying the association between muscle strength and mortality are not well understood. We were unable to ascertain whether the relation between muscle strength and mortality is direct or whether muscular strength is a surrogate marker of other factors underlying mortality. Future studies of interventions like resistance training, which has been shown to be efficacious in preserving muscular strength, ${ }^{23}$ could show whether maintenance of muscular strength translates into a reduction in mortality among weak elderly people. Our finding of increased risk for mortality among weak elderly people suggests that family physicians need to pay special attention to general prevention-related measures in these patients.

\section{Limitations}

Our study had limitations. Our assessment of comorbidities was limited to common chronic diseases. We did not address severity of disease, which could affect probability of survival. Lower handgrip strength can be an indicator of subclinical diseases that affect mortality. In addition, we were unable to adjust for the interim development or progression of comorbidities.

We did not observe an association between handgrip strength and cause-specific mortality, although several previous studies have linked poorer grip strength to mortality resulting from cardiovascular diseases, ${ }^{24,25}$ respiratory diseases ${ }^{25}$ and cancer. $^{24}$ This finding may be explained by the relatively small number of deaths from each specific cause in our study. The values for handgrip strength in our study population were comparable to other population-based studies involving older adults of similar ages. ${ }^{26}$ However, handgrip strength has been shown to be associated with ethnicity, and is weaker among Asian populations compared with Western. ${ }^{27}$ Given that our study was based on a homogenous Dutch population, the cutoff threshold for handgrip strength, below which is an increased risk for mortality, may not be generalizable to all elderly populations.

\section{Conclusion}

Our findings have substantial implications for care of a growing elderly population. Application of handgrip dynamometry as a screening tool in a multidimensional geriatric assessment may help identify older people at risk for disability and holds potential for use in prognostication of survival among elderly people. Further studies exploring factors that contribute to loss of muscular strength could provide valuable information toward the development of strategies for preserving muscular strength with advancing age.

This article has been peer reviewed.

Competing interest: None declared.

Contributors: Anton de Craen, Jacobijn Gussekloo and Rudi Westendorp were involved in the conceptual design of the study. Carolina Ling, Diana Taekema and Andrea Maier were involved in the analysis and interpretation of the data. Carolina Ling drafted the manuscript and wrote the final version. All of the authors critically reviewed the manuscript for important intellectual content and approved the final version submitted for publication. 
Acknowledgement: The authors thank Carolien Wijsman for her stimulating discussion and data analysis.

Funding: This study was supported by unrestricted grants from the Netherlands Organization of Scientific Research (ZonMw), the Ministry of Health, Welfare and Sports, and the Netherlands Genomics Initiative of the Netherlands Organization for Scientific Research.

\section{REFERENCES}

1. Wetle TF. The oldest old: missed public health opportunities. Am J Public Health 2008;98:1159.

2. World population ageing. 1950-2050. New York (NY): United Nations Publications; 2002. p. 23. Available: www.un.org/esa/population/publications/worldageing19502050/ (accessed 2009 Nov. 19).

3. Bauman AE. Updating the evidence that physical activity is good for health: an epidemiological review 2000-2003. J Sci Med Sport 2004;7 (Suppl 1):6-19.

4. Hu FB, Stampfer MJ, Colditz GA, et al. Physical activity and risk of stroke in women. JAMA 2000;283:2961-7.

5. Rolland Y, Czerwinski S, Abellan VK, et al. Sarcopenia: its assessment, etiology, pathogenesis, consequences and future perspectives. J Nutr Health Aging 2008; 12:433-50.

6. Lee JSW, Auyeung TW, Kwok T, et al. Associated factors and health impact of sarcopenia in older Chinese men and women: a cross-sectional study. Gerontology 2007;56:404-10.

7. Roubenoff R, Hughes VA. Sarcopenia: current concepts. J Gerontol A Biol Sci Med Sci 2000;55:M716-24.

8. Iannuzzi-Sucich M, Prestwood KM, Kenny AM. Prevalence of sarcopenia and predictors of skeletal muscle mass in healthy, older men and women. J Gerontol A Biol Sci Med Sci 2002;57:M772-7.

9. Giampaoli S, Ferrucci L, Cecchi F, et al. Hand-grip strength predicts incident disability in non-disabled older men. Age Ageing 1999;28:283-8.

10. Rantanen T, Avlund K, Suominen H, et al. Muscle strength as a predictor of onset of ADL dependence in people aged 75 years. Aging Clin Exp Res 2002;14(Suppl 3):10-5.

11. Alfaro-Acha A, Snih SA, Raji MA, et al. Handgrip strength and cognitive decline in older Mexican Americans. J Gerontol A Biol Sci Med Sci 2006;61:859-65.

12. Metter EJ, Talbot LA, Schrager M, et al. Skeletal muscle strength as a predictor of all-cause mortality in healthy men. J Gerontol A Biol Sci Med Sci 2002;57:B359-65.
13. Takata Y, Ansai T, Akifusa S, et al. Physical fitness and 4-year mortality in an 80year-old population. J Gerontol A Biol Sci Med Sci 2007;62:851-8.

14. Bohannon RW. Dynamometer measurements of hand-grip strength predict multiple outcomes. Percept Mot Skills 2001;93:323-8.

15. Innes E. Handgrip strength testing: a review of the literature. Aust Occup Ther J 1999;46:120-40.

16. Bohannon RW. Hand-grip dynamometry predicts future outcomes in aging adults J Geriatr Phys Ther 2008;31:3-10.

17. von Faber M, Bootsma-van der Wiel A, van Exel E, et al. Successful aging in the oldest old: Who can be characterized as successfully aged? Arch Intern Med 2001;161:2694-700.

18. Suurmeijer TP, Doeglas DM, Moum T, et al. The Groningen Activity Restriction scale for measuring disability: its utility in international comparisons. Am J Public Health 1994;84:1270-3.

19. van Eijk L. Activity and well-being in the elderly. Groningen (the Netherlands): University of Groningen; 1997.

20. Mosteller RD. Simplified calculation of body surface area. N Engl J Med 1987; 317:1098.

21. Nagelkerke NJD. A note on a general definition of the coefficient of determination. Biometrika 1991;73:691-2.

22. Martin HJ, Syddall HE, Dennison EM, et al. Relationship between customary physical activity, muscle strength and physical performance in older men and women: findings from the Hertfordshire Cohort Study. Age Ageing 2008;37:589-93.

23. Morey MC, Sloane R, Pieper CF, et al. Effect of physical activity guidelines on physical function in older adults. J Am Geriatr Soc 2008;56:1873-8.

24. Gale CR, Martyn CN, Cooper C, et al. Grip strength, body composition, and mortality. Int J Epidemiol 2007;36:228-35.

25. Rantanen T, Volpato S, Ferrucci L, et al. Handgrip strength and cause-specific and total mortality in older disabled women: exploring the mechanism. $J$ Am Geriatr Soc 2003;51:636-41.

26. Jansen CW, Niebuhr BR, Coussirat DJ, et al. Hand force of men and women over 65 years of age as measured by maximum pinch and grip force. J Aging Phys Act 2008;16:24-41.

27. Kamarul T, Ahmad TS, Loh WY. Hand grip strength in the adult Malaysian population. J Orthop Surg (Hong Kong) 2006;14:172-7.

Correspondence to: Carolina Ling, Leiden University Medical Center, Department of Gerontology and Geriatrics, Albinusdreef 2, 2333 ZA Leiden, Netherlands; c.h.y.ling@lumc.nl

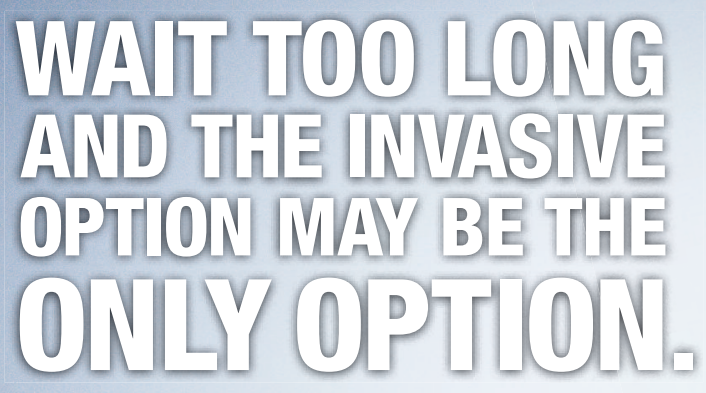

The wait-and-see approach can be a painful one for patients suffering from chronic neck or lower back pain, eventually making surgery the only option. If true non-surgical spinal decompression therapy is applied to suitable candidates early, long and costly surgical procedures and recovery periods can often be avoided.

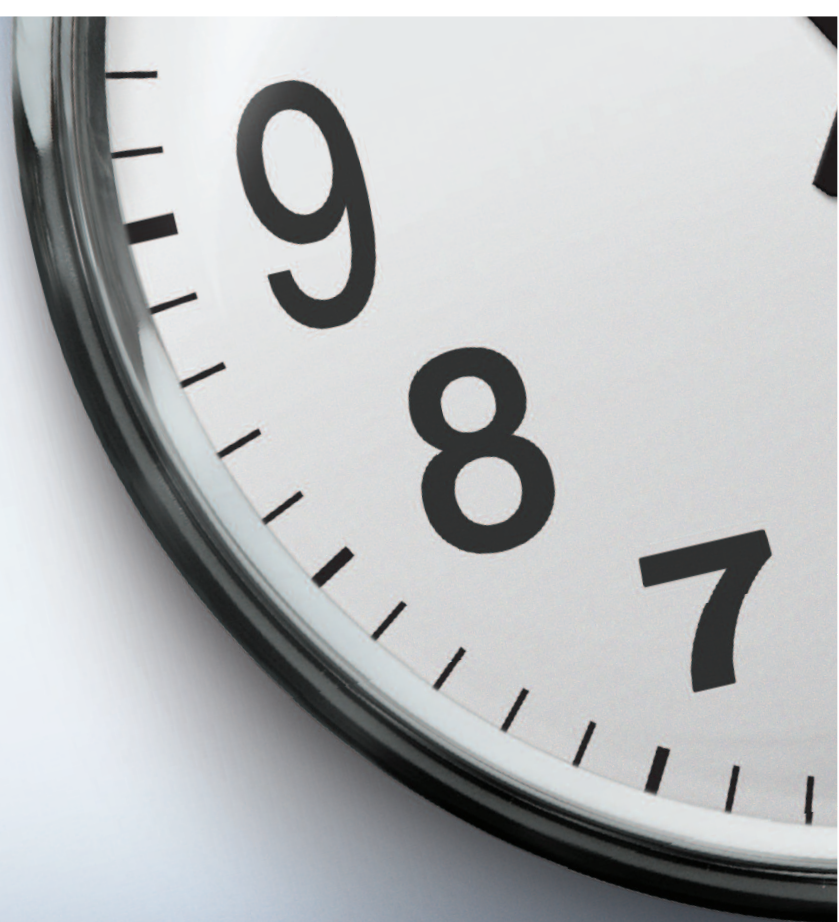

GET YOUR FREE DOGTOR KIT TODAY AT Www.spinal decompression kit.com Our free Information Kit will help you better determine which patients are good candidates for true non-surgical spinal decompression.

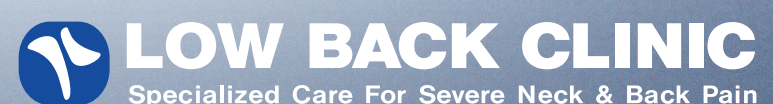

Specialized Care For Severe Neck \& Back Pain 